\title{
AARP
}

\section{Ageism Could Hurt Job Prospects, Say Job-Insecure Older Workers: Annotated Questionnaire, Ages 50-65 Only}

January 2021

Methodology: Survey was fielded to 1,502 adults ages 40-65 who are in the workforce. In addition, all respondents must have some job uncertainty over the next year, including at least one of the following: currently unemployed, need upskilling to keep their current job or get a new job, OR concerned that they could lose a job, be temporarily laid off, have hours reduced, or be furloughed.

Fielding dates were November 30 - December 7, 2020 via Dynata non-probability online panel and results were weighted to national benchmarks for workers ages 40-65. The following survey annotation includes results for workers ages 50-65 ( $n=861)$ and those results by age breaks.

For additional information, contact Rebecca Perron at rperron@aarp.org 


\section{ADDITIONAL SCREENING QUESTIONS}

Totals may not sum to $100 \%$ because of rounding or allowing multiple responses.

QS1. Which of the following best describes your current employment status?

\begin{tabular}{|c|c|c|c|}
\hline ALL & $\begin{array}{c}\text { NET } \\
\text { Ages 50-65 }\end{array}$ & Ages 50-59 & Ages $60-65$ \\
\hline Weighted Base & 861 & 623 & 237 \\
\hline $\begin{array}{l}\text { Working for } 35 \text { hours per week or more for } \\
\text { pay }\end{array}$ & $67 \%$ & $71 \%$ & $57 \%$ \\
\hline $\begin{array}{l}\text { Working for less than } 35 \text { hours per week for } \\
\text { pay }\end{array}$ & $18 \%$ & $16 \%$ & $24 \%$ \\
\hline Not working for pay but looking for work & $15 \%$ & $13 \%$ & $19 \%$ \\
\hline
\end{tabular}

QS2 (NET Ages 50-65) How concerned are you that each of the following may happen to you in the next year?

\begin{tabular}{|c|c|c|c|c|}
\hline EMPLOYED & $\begin{array}{c}\text { You could lose } \\
\text { your job }\end{array}$ & $\begin{array}{l}\text { You could be } \\
\text { furloughed from } \\
\text { your job. By } \\
\text { furlough, we } \\
\text { mean that - for } \\
\text { days or } \\
\text { sometimes } \\
\text { weeks - you are } \\
\text { not required to } \\
\text { work, and you } \\
\text { are not paid }\end{array}$ & $\begin{array}{c}\text { You could be } \\
\text { temporarily laid } \\
\text { off from your job }\end{array}$ & $\begin{array}{c}\text { Your employer } \\
\text { could reduce the } \\
\text { number of hours } \\
\text { you work }\end{array}$ \\
\hline Weighted Base & \multicolumn{4}{|c|}{733} \\
\hline Top 2 Box (Net) & $48 \%$ & $54 \%$ & $50 \%$ & $56 \%$ \\
\hline Very concerned (4) & $21 \%$ & $23 \%$ & $22 \%$ & $24 \%$ \\
\hline Somewhat concerned (3) & $27 \%$ & $31 \%$ & $29 \%$ & $32 \%$ \\
\hline Bottom 2 Box (Net) & $52 \%$ & $46 \%$ & $50 \%$ & $44 \%$ \\
\hline Not too concerned (2) & $33 \%$ & $28 \%$ & $31 \%$ & $26 \%$ \\
\hline Not at all concerned (1) & $19 \%$ & $18 \%$ & $19 \%$ & $18 \%$ \\
\hline
\end{tabular}




\section{AARP}

QS2 (AGES 50-59) How concerned are you that each of the following may happen to you in the next year?

\begin{tabular}{|c|c|c|c|c|}
\hline EMPLOYED & $\begin{array}{l}\text { You could lose } \\
\text { your job }\end{array}$ & $\begin{array}{l}\text { You could be } \\
\text { furloughed from } \\
\text { your job. By } \\
\text { furlough, we } \\
\text { mean that - for } \\
\text { days or } \\
\text { sometimes } \\
\text { weeks - you are } \\
\text { not required to } \\
\text { work, and you } \\
\text { are not paid }\end{array}$ & $\begin{array}{l}\text { You could be } \\
\text { temporarily laid } \\
\text { off from your job }\end{array}$ & $\begin{array}{c}\text { Your employer } \\
\text { could reduce the } \\
\text { number of hours } \\
\text { you work }\end{array}$ \\
\hline Weighted Base & \multicolumn{4}{|c|}{540} \\
\hline Top 2 Box (Net) & $50 \%$ & $56 \%$ & $52 \%$ & $59 \%$ \\
\hline Very concerned (4) & $22 \%$ & $26 \%$ & $24 \%$ & $28 \%$ \\
\hline Somewhat concerned (3) & $28 \%$ & $30 \%$ & $28 \%$ & $31 \%$ \\
\hline Bottom 2 Box (Net) & $50 \%$ & $45 \%$ & $48 \%$ & $42 \%$ \\
\hline Not too concerned (2) & $34 \%$ & $28 \%$ & $31 \%$ & $25 \%$ \\
\hline Not at all concerned (1) & $16 \%$ & $17 \%$ & $18 \%$ & $17 \%$ \\
\hline
\end{tabular}

QS2 (AGES 60-65) How concerned are you that each of the following may happen to you in the next year?

\begin{tabular}{|c|c|c|c|c|}
\hline EMPLOYED & $\begin{array}{l}\text { You could lose } \\
\text { your job }\end{array}$ & $\begin{array}{c}\text { You could be } \\
\text { furloughed from } \\
\text { your job. By } \\
\text { furlough, we } \\
\text { mean that - for } \\
\text { days or } \\
\text { sometimes } \\
\text { weeks - you are } \\
\text { not required to } \\
\text { work, and you } \\
\text { are not paid }\end{array}$ & $\begin{array}{l}\text { You could be } \\
\text { temporarily laid } \\
\text { off from your job }\end{array}$ & $\begin{array}{c}\text { Your employer } \\
\text { could reduce the } \\
\text { number of hours } \\
\text { you work }\end{array}$ \\
\hline Weighted Base & \multicolumn{4}{|c|}{193} \\
\hline Top 2 Box (Net) & $44 \%$ & $52 \%$ & $46 \%$ & $49 \%$ \\
\hline Very concerned (4) & $18 \%$ & $18 \%$ & $16 \%$ & $16 \%$ \\
\hline Somewhat concerned (3) & $26 \%$ & $34 \%$ & $30 \%$ & $33 \%$ \\
\hline Bottom 2 Box (Net) & $56 \%$ & $49 \%$ & $54 \%$ & $52 \%$ \\
\hline Not too concerned (2) & $32 \%$ & $29 \%$ & $31 \%$ & $30 \%$ \\
\hline Not at all concerned (1) & $24 \%$ & $20 \%$ & $24 \%$ & $22 \%$ \\
\hline
\end{tabular}




\section{AARP}

QS3. How important will it be for you to gain new skills in the next year so that you can keep your current job or find a new job?

\begin{tabular}{|c|c|c|c|}
\hline ALL & $\begin{array}{c}\text { NET } \\
\text { Ages 50-65 }\end{array}$ & Ages 50-59 & Ages $60-65$ \\
\hline Weighted Base & 861 & 623 & 237 \\
\hline Top 2 Box (Net) & $80 \%$ & $83 \%$ & $72 \%$ \\
\hline Very important (4) & $29 \%$ & $32 \%$ & $22 \%$ \\
\hline Somewhat important (3) & $51 \%$ & $51 \%$ & $50 \%$ \\
\hline Bottom 2 Box (Net) & $20 \%$ & $17 \%$ & $29 \%$ \\
\hline Not too important (2) & $15 \%$ & $13 \%$ & $19 \%$ \\
\hline Not at all important (1) & $5 \%$ & $4 \%$ & $10 \%$ \\
\hline
\end{tabular}

QS4a. Have you lost a job at any point during 2020?

\begin{tabular}{|l|c|c|c|}
\hline ALL & NET & Ages 50-59 & Ages 60-65 \\
\hline Weighted Base & Ages 50-65 & 623 & 237 \\
\hline Yes & 861 & $26 \%$ & $31 \%$ \\
\hline No & $27 \%$ & $74 \%$ & $69 \%$ \\
\hline
\end{tabular}

QS4b. How long has it been since you worked for pay?

\begin{tabular}{|c|c|c|c|}
\hline UNEMPLOYED & $\begin{array}{c}\text { NET } \\
\text { Ages } 50-65 \text { (Looking) }\end{array}$ & Ages 50-59 & Ages 60-65 \\
\hline Weighted Base & 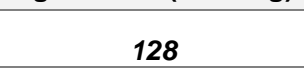 & Insufficient Base & Insufficient Base \\
\hline Less than one month & $2 \%$ & & \\
\hline One month to less than 3 months & $9 \%$ & & \\
\hline 3 months to less than 6 months & $11 \%$ & & \\
\hline 6 months to less than 9 months & $26 \%$ & & \\
\hline 9 months or more & $53 \%$ & & \\
\hline
\end{tabular}

QS5. How long have you worked in your current job?

\begin{tabular}{|c|c|c|c|}
\hline EMPLOYED & $\begin{array}{c}\text { NET } \\
\text { Ages 50-65 } \\
\text { EMPLOYED }\end{array}$ & Ages 50-59 & Ages 60-65 \\
\hline Weighted Base & 733 & 540 & 193 \\
\hline Less than 6 months & $5 \%$ & $5 \%$ & $4 \%$ \\
\hline 6 months to less than 1 year & $5 \%$ & $5 \%$ & $6 \%$ \\
\hline 1 year to less than 3 years & $12 \%$ & $13 \%$ & $11 \%$ \\
\hline 3 years to less than 6 years & $17 \%$ & $18 \%$ & $12 \%$ \\
\hline 6 years or more & $62 \%$ & $60 \%$ & $67 \%$ \\
\hline
\end{tabular}




\section{AARP}

\section{SURVEY}

Q1. (NET Ages 50-65) - Please indicate when the last time was that you took each of the following job steps:

\begin{tabular}{|l|c|c|c|c|}
\hline & $\begin{array}{c}\text { Searched for a } \\
\text { job }\end{array}$ & $\begin{array}{c}\text { Revised or } \\
\text { created a } \\
\text { resume }\end{array}$ & $\begin{array}{c}\text { Submitted a job } \\
\text { application }\end{array}$ & $\begin{array}{c}\text { Had a job } \\
\text { interview }\end{array}$ \\
\hline Weighted Base & \multicolumn{3}{|c|}{861} & $19 \%$ \\
\hline $\begin{array}{l}\text { In the past year } \\
\text { More than 1 year ago but less than 3 years } \\
\text { ago }\end{array}$ & $36 \%$ & $33 \%$ & $16 \%$ & $17 \%$ \\
\hline $\begin{array}{l}\text { More than 3 years ago but less than 5 years } \\
\text { ago }\end{array}$ & $14 \%$ & $17 \%$ & $11 \%$ & $15 \%$ \\
\hline More than 5 years ago & $40 \%$ & $34 \%$ & $44 \%$ & $49 \%$ \\
\hline
\end{tabular}

Q1. (AGES 50-59) - Please indicate when the last time was that you took each of the following job steps:

\begin{tabular}{|c|c|c|c|c|}
\hline ALL & $\begin{array}{c}\text { Searched for a } \\
\text { job }\end{array}$ & $\begin{array}{l}\text { Revised or } \\
\text { created a } \\
\text { resume }\end{array}$ & $\begin{array}{l}\text { Submitted a job } \\
\text { application }\end{array}$ & $\begin{array}{l}\text { Had a job } \\
\text { interview }\end{array}$ \\
\hline Weighted Base & \multicolumn{4}{|c|}{623} \\
\hline In the past year & $36 \%$ & $34 \%$ & $28 \%$ & $20 \%$ \\
\hline $\begin{array}{l}\text { More than } 1 \text { year ago but less than } 3 \text { years } \\
\text { ago }\end{array}$ & $14 \%$ & $18 \%$ & $17 \%$ & $16 \%$ \\
\hline $\begin{array}{l}\text { More than } 3 \text { years ago but less than } 5 \text { years } \\
\text { ago }\end{array}$ & $11 \%$ & $15 \%$ & $13 \%$ & $16 \%$ \\
\hline More than 5 years ago & $39 \%$ & $33 \%$ & $42 \%$ & $48 \%$ \\
\hline
\end{tabular}

Q1. (AGES 60-65) - Please indicate when the last time was that you took each of the following job steps:

\begin{tabular}{|c|c|c|c|c|}
\hline ALL & $\begin{array}{l}\text { Searched for a } \\
\text { job }\end{array}$ & $\begin{array}{l}\text { Revised or } \\
\text { created a } \\
\text { resume }\end{array}$ & $\begin{array}{l}\text { Submitted a job } \\
\text { application }\end{array}$ & $\begin{array}{l}\text { Had a job } \\
\text { interview }\end{array}$ \\
\hline Weighted Base & \multicolumn{4}{|c|}{237} \\
\hline In the past year & $37 \%$ & $32 \%$ & $32 \%$ & $16 \%$ \\
\hline $\begin{array}{l}\text { More than } 1 \text { year ago but less than } 3 \text { years } \\
\text { ago }\end{array}$ & $13 \%$ & $15 \%$ & $14 \%$ & $20 \%$ \\
\hline $\begin{array}{l}\text { More than } 3 \text { years ago but less than } 5 \text { years } \\
\text { ago }\end{array}$ & $7 \%$ & $14 \%$ & $7 \%$ & $11 \%$ \\
\hline More than 5 years ago & $43 \%$ & $39 \%$ & $47 \%$ & $53 \%$ \\
\hline
\end{tabular}




\section{AARP}

Q2. (NET Ages 50-65) - How prepared do you feel you are about each of the following?

\begin{tabular}{|c|c|c|c|c|}
\hline ALL & $\begin{array}{l}\text { Knowing where } \\
\text { to look for a job }\end{array}$ & $\begin{array}{c}\text { Knowing how to } \\
\text { use your } \\
\text { network to find } \\
\text { job } \\
\text { opportunities }\end{array}$ & $\begin{array}{c}\text { Knowing what } \\
\text { you need to do } \\
\text { to update your } \\
\text { resume }\end{array}$ & $\begin{array}{l}\text { Knowing how to } \\
\text { prepare for an } \\
\text { interview }\end{array}$ \\
\hline Weighted Base & \multicolumn{4}{|c|}{861} \\
\hline Top 2 Box (Net) & $78 \%$ & $70 \%$ & $75 \%$ & $79 \%$ \\
\hline Very prepared (4) & $31 \%$ & $27 \%$ & $32 \%$ & $31 \%$ \\
\hline Somewhat prepared (3) & $47 \%$ & $44 \%$ & $43 \%$ & $48 \%$ \\
\hline Bottom 2 Box (Net) & $22 \%$ & $30 \%$ & $25 \%$ & $21 \%$ \\
\hline Not too prepared (2) & $17 \%$ & $21 \%$ & $19 \%$ & $16 \%$ \\
\hline Not at all prepared (1) & $6 \%$ & $9 \%$ & $6 \%$ & $5 \%$ \\
\hline
\end{tabular}

Q2. (NET Ages 50-65) - How prepared do you feel you are about each of the following?

\begin{tabular}{|c|c|c|c|c|}
\hline ALL & $\begin{array}{l}\text { Knowing how to } \\
\text { use technology } \\
\text { for a video } \\
\text { interview (i.e. } \\
\text { using Zoom or } \\
\text { Skype) }\end{array}$ & $\begin{array}{l}\text { Knowing how to } \\
\text { negotiate a job } \\
\text { offer/salary offer }\end{array}$ & $\begin{array}{l}\text { Knowing how to } \\
\text { age-proof your } \\
\text { resume, } \\
\text { including } \\
\text { removing dates } \\
\text { that can be used } \\
\text { to determine } \\
\text { your age }\end{array}$ & $\begin{array}{l}\text { Knowing how to } \\
\text { improve your } \\
\text { online presence } \\
\text { (e.g. personal } \\
\text { website, } \\
\text { Linkedln profile) }\end{array}$ \\
\hline Weighted Base & \multicolumn{4}{|c|}{861} \\
\hline Top 2 Box (Net) & $69 \%$ & $67 \%$ & $57 \%$ & $61 \%$ \\
\hline Very prepared (4) & $35 \%$ & $26 \%$ & $24 \%$ & $21 \%$ \\
\hline Somewhat prepared (3) & $34 \%$ & $41 \%$ & $34 \%$ & $40 \%$ \\
\hline Bottom 2 Box (Net) & $31 \%$ & $33 \%$ & $43 \%$ & $39 \%$ \\
\hline Not too prepared (2) & $21 \%$ & $24 \%$ & $29 \%$ & $26 \%$ \\
\hline Not at all prepared (1) & $10 \%$ & $9 \%$ & $14 \%$ & $13 \%$ \\
\hline
\end{tabular}

Q2. (AGES 50-59) - How prepared do you feel you are about each of the following?

\begin{tabular}{|c|c|c|c|c|}
\hline ALL & $\begin{array}{l}\text { Knowing where } \\
\text { to look for a job }\end{array}$ & $\begin{array}{c}\text { Knowing how } \\
\text { to use your } \\
\text { network to find } \\
\text { job } \\
\text { opportunities }\end{array}$ & $\begin{array}{l}\text { Knowing what } \\
\text { you need to do to } \\
\text { update your } \\
\text { resume }\end{array}$ & $\begin{array}{l}\text { Knowing how } \\
\text { to prepare for } \\
\text { an interview }\end{array}$ \\
\hline Weighted Base & \multicolumn{4}{|c|}{623} \\
\hline Top 2 Box (Net) & $79 \%$ & $72 \%$ & $76 \%$ & $79 \%$ \\
\hline Very prepared (4) & $31 \%$ & $27 \%$ & $33 \%$ & $31 \%$ \\
\hline Somewhat prepared (3) & $48 \%$ & $45 \%$ & $43 \%$ & $48 \%$ \\
\hline
\end{tabular}




\section{AARP}

\begin{tabular}{|l|c|c|c|c|}
\hline Bottom 2 Box (Net) & $21 \%$ & $29 \%$ & $25 \%$ & $21 \%$ \\
\hline Not too prepared (2) & $16 \%$ & $19 \%$ & $18 \%$ & $15 \%$ \\
\hline Not at all prepared (1) & $6 \%$ & $9 \%$ & $7 \%$ & $6 \%$ \\
\hline
\end{tabular}

\section{Q2. (AGES 50-59) - How prepared do you feel you are about each of the following?}

\begin{tabular}{|c|c|c|c|c|}
\hline ALL & $\begin{array}{l}\text { Knowing how to } \\
\text { use technology } \\
\text { for a video } \\
\text { interview (i.e. } \\
\text { using Zoom or } \\
\text { Skype) }\end{array}$ & $\begin{array}{l}\text { Knowing how to } \\
\text { negotiate a job } \\
\text { offer/salary offer }\end{array}$ & $\begin{array}{l}\text { Knowing how to } \\
\text { age-proof your } \\
\text { resume, } \\
\text { including } \\
\text { removing dates } \\
\text { that can be used } \\
\text { to determine } \\
\text { your age }\end{array}$ & $\begin{array}{l}\text { Knowing how to } \\
\text { improve your } \\
\text { online presence } \\
\text { (e.g. personal } \\
\text { website, } \\
\text { Linkedln profile) }\end{array}$ \\
\hline Weighted Base & \multicolumn{4}{|c|}{623} \\
\hline Top 2 Box (Net) & $70 \%$ & $66 \%$ & $58 \%$ & $61 \%$ \\
\hline Very prepared (4) & $36 \%$ & $26 \%$ & $23 \%$ & $21 \%$ \\
\hline Somewhat prepared (3) & $34 \%$ & $40 \%$ & $35 \%$ & $40 \%$ \\
\hline Bottom 2 Box (Net) & $30 \%$ & $34 \%$ & $42 \%$ & $39 \%$ \\
\hline Not too prepared (2) & $20 \%$ & $26 \%$ & $29 \%$ & $26 \%$ \\
\hline Not at all prepared (1) & $10 \%$ & $8 \%$ & $13 \%$ & $13 \%$ \\
\hline
\end{tabular}

Q2. (AGES 60-65) - How prepared do you feel you are about each of the following?

\begin{tabular}{|c|c|c|c|c|}
\hline ALL & $\begin{array}{l}\text { Knowing where } \\
\text { to look for a job }\end{array}$ & $\begin{array}{c}\text { Knowing how to } \\
\text { use your } \\
\text { network to find } \\
\text { job } \\
\text { opportunities }\end{array}$ & $\begin{array}{c}\text { Knowing what } \\
\text { you need to do } \\
\text { to update your } \\
\text { resume }\end{array}$ & $\begin{array}{c}\text { Knowing how to } \\
\text { prepare for an } \\
\text { interview }\end{array}$ \\
\hline Weighted Base & \multicolumn{4}{|c|}{237} \\
\hline Top 2 Box (Net) & $74 \%$ & $68 \%$ & $73 \%$ & $79 \%$ \\
\hline Very prepared (4) & $30 \%$ & $27 \%$ & $29 \%$ & $31 \%$ \\
\hline Somewhat prepared (3) & $44 \%$ & $40 \%$ & $44 \%$ & $47 \%$ \\
\hline Bottom 2 Box (Net) & $26 \%$ & $33 \%$ & $27 \%$ & $22 \%$ \\
\hline Not too prepared (2) & $20 \%$ & $26 \%$ & $22 \%$ & $19 \%$ \\
\hline Not at all prepared (1) & $6 \%$ & $7 \%$ & $5 \%$ & $3 \%$ \\
\hline
\end{tabular}




\section{$\triangle A R P^{\circ}$}

Q2. (AGES 60-65) - How prepared do you feel you are about each of the following?

\begin{tabular}{|c|c|c|c|c|}
\hline ALL & $\begin{array}{l}\text { Knowing how to } \\
\text { use technology } \\
\text { for a video } \\
\text { interview (i.e. } \\
\text { using Zoom or } \\
\text { Skype) }\end{array}$ & $\begin{array}{l}\text { Knowing how to } \\
\text { negotiate a job } \\
\text { offer/salary offer }\end{array}$ & $\begin{array}{l}\text { Knowing how to } \\
\text { age-proof your } \\
\text { resume, } \\
\text { including } \\
\text { removing dates } \\
\text { that can be used } \\
\text { to determine } \\
\text { your age }\end{array}$ & $\begin{array}{l}\text { Knowing how to } \\
\text { improve your } \\
\text { online presence } \\
\text { (e.g. personal } \\
\text { website, } \\
\text { Linkedln profile) }\end{array}$ \\
\hline Weighted Base & \multicolumn{4}{|c|}{237} \\
\hline Top 2 Box (Net) & $66 \%$ & $68 \%$ & $54 \%$ & $61 \%$ \\
\hline Very prepared (4) & $31 \%$ & $25 \%$ & $24 \%$ & $20 \%$ \\
\hline Somewhat prepared (3) & $35 \%$ & $43 \%$ & $30 \%$ & $41 \%$ \\
\hline Bottom 2 Box (Net) & $34 \%$ & $32 \%$ & $46 \%$ & $39 \%$ \\
\hline Not too prepared (2) & $23 \%$ & $20 \%$ & $29 \%$ & $26 \%$ \\
\hline Not at all prepared (1) & $11 \%$ & $12 \%$ & $17 \%$ & $13 \%$ \\
\hline
\end{tabular}

Q3. How likely is it that you would have to learn new skills in order to get a new job?

\begin{tabular}{|l|c|c|c|}
\hline \multirow{2}{*}{ ALL } & $\begin{array}{c}\text { NET } \\
\text { Ages 50-65 }\end{array}$ & Ages 50-59 & Ages 60-65 \\
\hline Weighted Base & $\mathbf{8 6 1}$ & $\mathbf{6 2 3}$ & $\mathbf{2 3 7}$ \\
\hline Top 2 Box (Net) & $69 \%$ & $71 \%$ & $15 \%$ \\
\hline Very likely (4) & $19 \%$ & $21 \%$ & $50 \%$ \\
\hline Somewhat likely (3) & $50 \%$ & $50 \%$ & $35 \%$ \\
\hline Bottom 2 Box (Net) & $31 \%$ & $29 \%$ & $28 \%$ \\
\hline Not too likely (2) & $25 \%$ & $23 \%$ & $7 \%$ \\
\hline Not at all likely (1) & $6 \%$ & $6 \%$ & \\
\hline
\end{tabular}

Q4. Please select which of the following feelings you have as you think about the job search process.

\begin{tabular}{|l|c|c|c|}
\hline ALL & $\begin{array}{c}\text { NET } \\
\text { Ages 50-65 }\end{array}$ & Ages 50-59 & Ages 60-65 \\
\hline Weighted Base & 861 & 623 & $32 \%$ \\
\hline Hopeful & $36 \%$ & $38 \%$ & $33 \%$ \\
\hline Nervous & $35 \%$ & $36 \%$ & $23 \%$ \\
\hline Positive & $28 \%$ & $30 \%$ & $25 \%$ \\
\hline Optimistic & $31 \%$ & $33 \%$ & $26 \%$ \\
\hline Confident & $28 \%$ & $28 \%$ & $21 \%$ \\
\hline Overwhelmed & $23 \%$ & $24 \%$ & \\
\hline
\end{tabular}




\begin{tabular}{|l|c|c|c|}
\hline Prepared & $22 \%$ & $23 \%$ & $19 \%$ \\
\hline Frustrated & $22 \%$ & $22 \%$ & $22 \%$ \\
\hline Excited & $16 \%$ & $20 \%$ & $8 \%$ \\
\hline Fearful & $18 \%$ & $18 \%$ & $18 \%$ \\
\hline Pessimistic & $15 \%$ & $13 \%$ & $20 \%$ \\
\hline Unprepared & $13 \%$ & $13 \%$ & $14 \%$ \\
\hline Annoyed & $11 \%$ & $11 \%$ & $11 \%$ \\
\hline Hopeless & $9 \%$ & $9 \%$ & $9 \%$ \\
\hline Sad & $8 \%$ & $8 \%$ & $9 \%$ \\
\hline Negative & $9 \%$ & $9 \%$ & $5 \%$ \\
\hline Confused & $7 \%$ & $8 \%$ & $11 \%$ \\
\hline None of the above & $7 \%$ & $6 \%$ & $4 \%$ \\
\hline Invigorated & $6 \%$ & $6 \%$ & \\
\hline
\end{tabular}

Q5. Which of the following feelings is how you feel most of the time as you consider the job search process?

\begin{tabular}{|c|c|c|c|}
\hline ALL & $\begin{array}{c}\text { NET } \\
\text { Ages 50-65Selected at } \\
\text { least one emotion }\end{array}$ & Ages 50-59 & Ages 60-65 \\
\hline Weighted Base & 800 & 588 & 212 \\
\hline Nervous & $14 \%$ & $15 \%$ & $12 \%$ \\
\hline Hopeful & $12 \%$ & $11 \%$ & $14 \%$ \\
\hline Optimistic & $12 \%$ & $12 \%$ & $10 \%$ \\
\hline Confident & $11 \%$ & $11 \%$ & $12 \%$ \\
\hline Overwhelmed & $9 \%$ & $9 \%$ & $10 \%$ \\
\hline Positive & $6 \%$ & $6 \%$ & $6 \%$ \\
\hline Excited & $4 \%$ & $4 \%$ & $2 \%$ \\
\hline Frustrated & $7 \%$ & $6 \%$ & $10 \%$ \\
\hline Prepared & $6 \%$ & $6 \%$ & $3 \%$ \\
\hline Unprepared & $5 \%$ & $5 \%$ & $6 \%$ \\
\hline Pessimistic & $4 \%$ & $4 \%$ & $6 \%$ \\
\hline Annoyed & $2 \%$ & $2 \%$ & $3 \%$ \\
\hline Hopeless & $2 \%$ & $2 \%$ & $3 \%$ \\
\hline Fearful & $2 \%$ & $3 \%$ & $2 \%$ \\
\hline Negative & $1 \%$ & $1 \%$ & $1 \%$ \\
\hline Invigorated & $1 \%$ & $1 \%$ & $1 \%$ \\
\hline
\end{tabular}




\begin{tabular}{|l|c|c|c|}
\hline Sad & $1 \%$ & $1 \%$ & $2 \%$ \\
\hline Confused & $1 \%$ & $1 \%$ & $1 \%$ \\
\hline
\end{tabular}

Q6. (NET Ages 50-65) - Which of the following are reasons that you lost or left your most recent job?

\begin{tabular}{|c|c|c|c|c|}
\hline UNEMPLOYED & $\begin{array}{c}\text { The Covid-19 } \\
\text { pandemic }\end{array}$ & $\begin{array}{l}\text { Discrimination } \\
\text { due to my age }\end{array}$ & $\begin{array}{l}\text { The company } \\
\text { was downsizing }\end{array}$ & $\begin{array}{l}\text { I needed to stay } \\
\text { home with my } \\
\text { children (either } \\
\text { for child care } \\
\text { reasons or } \\
\text { remote } \\
\text { schooling) }\end{array}$ \\
\hline Weighted Base & \multicolumn{4}{|c|}{128} \\
\hline A major reason & $43 \%$ & $12 \%$ & $25 \%$ & $2 \%$ \\
\hline A minor reason & $15 \%$ & $17 \%$ & $15 \%$ & $7 \%$ \\
\hline Not a reason & $42 \%$ & $71 \%$ & $60 \%$ & $92 \%$ \\
\hline
\end{tabular}

Q6. (NET Ages 50-65) - Which of the following are reasons that you lost or left your most recent job?

\begin{tabular}{|l|c|c|c|c|}
\hline & $\begin{array}{c}\text { The company } \\
\text { closed due to } \\
\text { Covid }\end{array}$ & $\begin{array}{c}\text { Discrimination } \\
\text { due to my race } \\
\text { or ethnicity }\end{array}$ & $\begin{array}{c}\text { I was worried } \\
\text { about my health } \\
\text { because of } \\
\text { Covid }\end{array}$ & $\begin{array}{c}\text { The company is } \\
\text { trying save } \\
\text { money }\end{array}$ \\
\hline Weighted Base & \multicolumn{3}{|c|}{$\mathbf{1 2 8}$} & \\
\hline A major reason & $28 \%$ & $7 \%$ & $21 \%$ & $23 \%$ \\
\hline A minor reason & $6 \%$ & $7 \%$ & $13 \%$ & $19 \%$ \\
\hline Not a reason & $65 \%$ & $87 \%$ & $66 \%$ & $58 \%$ \\
\hline
\end{tabular}

Q6. (AGES 50-59) - Which of the following are reasons that you lost or left your most recent job?

\begin{tabular}{|l|l|l|l|l|}
\hline & & & \multicolumn{2}{|c|}{$\begin{array}{c}\text { I needed to stay } \\
\text { home with my } \\
\text { children (either } \\
\text { for child care } \\
\text { reasons or } \\
\text { remote } \\
\text { schooling) }\end{array}$} \\
\hline UNEMPLOYED & $\begin{array}{c}\text { The Covid-19 } \\
\text { pandemic }\end{array}$ & $\begin{array}{c}\text { Discrimination } \\
\text { due to my age }\end{array}$ & $\begin{array}{c}\text { The company } \\
\text { was downsizing }\end{array}$ & $\begin{array}{c}\text { Insufficient Base } \\
\text { Weighted Base }\end{array}$ \\
\hline A major reason & & & & \\
\hline A minor reason & & & & \\
\hline Not a reason & & & & \\
\hline
\end{tabular}




\section{$\triangle A R P^{\circ}$}

Q6. (AGES 50-59) - Which of the following are reasons that you lost or left your most recent job?

\begin{tabular}{|c|c|c|c|c|}
\hline UNEMPLOYED & $\begin{array}{c}\text { The company } \\
\text { closed due to } \\
\text { Covid }\end{array}$ & $\begin{array}{l}\text { Discrimination } \\
\text { due to my race } \\
\text { or ethnicity }\end{array}$ & $\begin{array}{l}\text { I was worried } \\
\text { about my health } \\
\text { because of } \\
\text { Covid }\end{array}$ & $\begin{array}{c}\text { The company is } \\
\text { trying to save } \\
\text { money }\end{array}$ \\
\hline Weighted Base & \multicolumn{4}{|c|}{ Insufficient Base } \\
\hline A major reason & & & & \\
\hline A minor reason & & & & \\
\hline Not a reason & & & & \\
\hline
\end{tabular}

Q6. (AGES 60-65) - Which of the following are reasons that you lost or left your most recent job?

\begin{tabular}{|l|l|l|l|l|}
\hline & & \multicolumn{2}{|c|}{} & $\begin{array}{c}\text { I needed to stay } \\
\text { home with my } \\
\text { children (either } \\
\text { for child care } \\
\text { reasons or } \\
\text { remote } \\
\text { schooling) }\end{array}$ \\
\hline UNEMPLOYED & $\begin{array}{c}\text { The Covid-19 } \\
\text { pandemic }\end{array}$ & $\begin{array}{c}\text { Discrimination } \\
\text { due to my age }\end{array}$ & $\begin{array}{c}\text { The company } \\
\text { was downsizing }\end{array}$ & $\begin{array}{c}\text { Insufficient Base } \\
\text { Weighted Base }\end{array}$ \\
\hline A major reason & & & & \\
\hline A minor reason & & & & \\
\hline Not a reason & & & & \\
\hline
\end{tabular}

Q6. (AGES 60-65) - Which of the following are reasons that you lost or left your most recent job?

\begin{tabular}{|l|l|l|l|l|}
\hline & $\begin{array}{c}\text { The company } \\
\text { closed due to } \\
\text { Covid }\end{array}$ & $\begin{array}{c}\text { Discrimination } \\
\text { due to my race } \\
\text { or ethnicity }\end{array}$ & $\begin{array}{c}\text { I was worried } \\
\text { about my health } \\
\text { because of } \\
\text { Covid }\end{array}$ & $\begin{array}{c}\text { The company is } \\
\text { trying to save } \\
\text { money }\end{array}$ \\
\hline Weighted Base & \multicolumn{3}{|c|}{ Insufficient Base } \\
\hline A major reason & & & & \\
\hline A minor reason & & & & \\
\hline Not a reason & & & \\
\hline
\end{tabular}

Q6a. How confident are you that you will get another job within the next three months?

\begin{tabular}{|l|c|c|c|}
\hline & $\begin{array}{c}\text { NET } \\
\text { Uges 50-65 } \\
\text { UNEMPLOYED }\end{array}$ & Ages 50-59 & Ages 60-65 \\
\hline Weighted Base & 128 & Insufficient base & Insufficient base \\
\hline Top 2 Box (Net) & $49 \%$ & & \\
\hline Very confident (4) & $16 \%$ & & \\
\hline
\end{tabular}




\section{A ARP}

\begin{tabular}{|l|c|l|}
\hline Somewhat confident (3) & $33 \%$ & \\
\hline Bottom 2 Box (Net) & $50 \%$ & \\
\hline Not too confident (2) & $36 \%$ & \\
\hline Not at all confident (1) & $14 \%$ & \\
\hline
\end{tabular}

Q7. (NET Ages 50-65) - Which of the following are reasons that you are concerned that you could lose or leave your job in the next year?

\begin{tabular}{|c|c|c|c|c|}
\hline $\begin{array}{l}\text { FEAR COULD LOSE/LEAVE JOB IN } \\
\text { NEXT YEAR }\end{array}$ & $\begin{array}{l}\text { The Covid-19 } \\
\text { pandemic }\end{array}$ & $\begin{array}{l}\text { Discrimination } \\
\text { due to my age }\end{array}$ & $\begin{array}{l}\text { The company } \\
\text { may downsize }\end{array}$ & $\begin{array}{l}\text { I need to stay } \\
\text { home with my } \\
\text { children (either } \\
\text { for child care } \\
\text { reasons or } \\
\text { remote } \\
\text { schooling) }\end{array}$ \\
\hline Weighted Base & \multicolumn{4}{|c|}{353} \\
\hline A major reason & $62 \%$ & $29 \%$ & $41 \%$ & $10 \%$ \\
\hline A minor reason & $25 \%$ & $35 \%$ & $38 \%$ & $11 \%$ \\
\hline Not a reason & $13 \%$ & $36 \%$ & $21 \%$ & $79 \%$ \\
\hline
\end{tabular}

Q7. (NET Ages 50-65) - Which of the following are reasons that you are concerned that you could lose or leave your job in the next year?

\begin{tabular}{|l|c|c|c|}
\hline $\begin{array}{l}\text { FEAR COULD LOSE/LEAVE JOB IN THE } \\
\text { NEXT YEAR }\end{array}$ & $\begin{array}{c}|c| \\
\text { The company may } \\
\text { close due to Covid }\end{array}$ & $\begin{array}{c}\text { Discrimination due to } \\
\text { my race or ethnicity }\end{array}$ & $\begin{array}{c}\text { I meed to leave } \\
\text { because of concern } \\
\text { about my health } \\
\text { because of Covid }\end{array}$ \\
\hline Weighted Base & \multicolumn{3}{|c|}{697} \\
\hline A major reason & $40 \%$ & $9 \%$ & $25 \%$ \\
\hline A minor reason & $31 \%$ & $18 \%$ & $34 \%$ \\
\hline Not a reason & $29 \%$ & $74 \%$ & $41 \%$ \\
\hline
\end{tabular}

Q7. (AGES 50-59) - Which of the following are reasons that you are concerned that you could lose or leave your job in the next year?

\begin{tabular}{|c|c|c|c|c|}
\hline $\begin{array}{l}\text { FEAR COULD LOSE/LEAVE JOB IN } \\
\text { NEXT YEAR }\end{array}$ & $\begin{array}{l}\text { The Covid-19 } \\
\text { pandemic }\end{array}$ & $\begin{array}{l}\text { Discrimination } \\
\text { due to my age }\end{array}$ & $\begin{array}{l}\text { The company } \\
\text { may downsize }\end{array}$ & $\begin{array}{l}\text { I need to stay } \\
\text { home with my } \\
\text { children (either } \\
\text { for child care } \\
\text { reasons or } \\
\text { remote } \\
\text { schooling) }\end{array}$ \\
\hline Weighted Base & \multicolumn{4}{|c|}{268} \\
\hline A major reason & $62 \%$ & $26 \%$ & $44 \%$ & $12 \%$ \\
\hline A minor reason & $24 \%$ & $36 \%$ & $36 \%$ & $12 \%$ \\
\hline
\end{tabular}




\section{AARP}

Not a reason

$14 \%$

$37 \%$

$19 \%$

$77 \%$

Q7. (AGES 50-59) - Which of the following are reasons that you are concerned that you could lose or leave your job in the next year?

\begin{tabular}{|l|c|c|c|}
\hline $\begin{array}{l}\text { FEAR COULD LOSE/LEAVE JOB IN } \\
\text { NEXT YEAR }\end{array}$ & $\begin{array}{c}\text { The company may } \\
\text { close due to Covid }\end{array}$ & $\begin{array}{c}\text { Discrimination due to } \\
\text { my race or ethnicity }\end{array}$ & $\begin{array}{c}\text { I may need to leave } \\
\text { because of concern } \\
\text { about my health } \\
\text { because of Covid }\end{array}$ \\
\hline Weighted Base & \multicolumn{2}{|c|}{268} & $26 \%$ \\
\hline A major reason & $40 \%$ & $8 \%$ & $32 \%$ \\
\hline A minor reason & $30 \%$ & $18 \%$ & $43 \%$ \\
\hline Not a reason & $29 \%$ & $74 \%$ & \\
\hline
\end{tabular}

Q7. (AGES 60-65) - Which of the following are reasons that you are concerned that you could lose or leave your job in the next year?

\begin{tabular}{|c|c|c|c|c|}
\hline $\begin{array}{l}\text { FEAR COULD LOSE/LEAVE JOB IN } \\
\text { NEXT YEAR }\end{array}$ & $\begin{array}{l}\text { The Covid-19 } \\
\text { pandemic }\end{array}$ & $\begin{array}{l}\text { Discrimination } \\
\text { due to my age }\end{array}$ & $\begin{array}{l}\text { The company } \\
\text { may downsize }\end{array}$ & $\begin{array}{l}\text { I need to stay } \\
\text { home with my } \\
\text { children (either } \\
\text { for child care } \\
\text { reasons or } \\
\text { remote } \\
\text { schooling) }\end{array}$ \\
\hline Weighted Base & \multicolumn{4}{|c|}{ Insufficient Base } \\
\hline A major reason & & & & \\
\hline \multicolumn{5}{|l|}{ A minor reason } \\
\hline Not a reason & & & & \\
\hline
\end{tabular}

Q7. (AGES 60-65) - Which of the following are reasons that you are concerned that you could lose or leave your job in the next year?

\begin{tabular}{|l|l|l|l|}
\hline $\begin{array}{l}\text { FEAR COULD LOSE/LEAVE JOB IN } \\
\text { NEXT YEAR }\end{array}$ & $\begin{array}{l}\text { The company may may need to leave } \\
\text { close due to Covid }\end{array}$ & $\begin{array}{c}\text { I } \\
\text { Decause of concern } \\
\text { my race or ethnicity } \\
\text { about my health } \\
\text { because of Covid }\end{array}$ \\
\hline Weighted Base & & \\
\hline A major reason & & \\
\hline A minor reason & & \\
\hline Not a reason & & \\
\hline
\end{tabular}




\section{AARP}

Q8. Has your employer ever told you that you had to either change your work schedule, leave work, or not return to work because they are concerned about how your age may impact getting Covid-19?

\begin{tabular}{|l|c|c|c|}
\hline & NET & Ages 50-59 & Ages 60-65 \\
\hline WLL & Ages 50-65 & & \\
\hline Yes & 861 & 623 & 237 \\
\hline No & $15 \%$ & $12 \%$ & $80 \%$ \\
\hline
\end{tabular}

Q9. Have you considered or done any of the following because of Covid?

\begin{tabular}{|c|c|c|c|}
\hline ALL & $\begin{array}{c}\text { NET } \\
\text { Ages 50-65 }\end{array}$ & Ages 50-59 & Ages 60-65 \\
\hline Weighted Base & 861 & 623 & 237 \\
\hline $\begin{array}{l}\text { Leaving my current job because I am } \\
\text { concerned about being exposed to Covid }\end{array}$ & $8 \%$ & $8 \%$ & $8 \%$ \\
\hline $\begin{array}{l}\text { Changing careers so that I am not exposed } \\
\text { to Covid }\end{array}$ & $7 \%$ & $8 \%$ & $4 \%$ \\
\hline $\begin{array}{l}\text { Getting new skills or new degree to get a } \\
\text { job that allows me to work from home }\end{array}$ & $15 \%$ & $15 \%$ & $16 \%$ \\
\hline Retiring earlier than previously planned & $16 \%$ & $14 \%$ & $21 \%$ \\
\hline Retiring later than previous planned. & $9 \%$ & $8 \%$ & $13 \%$ \\
\hline None of the above & $60 \%$ & $63 \%$ & $55 \%$ \\
\hline
\end{tabular}

Q10. Suppose you (or your spouse) lost your income suddenly. For how many months would you be able to continue your current lifestyle if you had to rely only on your (and your spouse) savings, without withdrawing extra money from retirement accounts?

\begin{tabular}{|l|c|c|c|}
\hline \multicolumn{1}{|l|}{ ALL } & $\begin{array}{c}\text { NET } \\
\text { Ages 50-65 }\end{array}$ & Ages 50-59 & Ages 60-65 \\
\hline Weighted Base & $\mathbf{8 6 1}$ & $\mathbf{6 2 3}$ & $\mathbf{2 3 7}$ \\
\hline 1 month or less & $17 \%$ & $19 \%$ & $12 \%$ \\
\hline 2 months & $12 \%$ & $12 \%$ & $16 \%$ \\
\hline 3 months & $18 \%$ & $18 \%$ & $5 \%$ \\
\hline 4 months & $7 \%$ & $8 \%$ & $2 \%$ \\
\hline 5 months & $4 \%$ & $5 \%$ & $51 \%$ \\
\hline 6 months or more & $42 \%$ & $39 \%$ & $5 \%$ \\
\hline
\end{tabular}




\section{AARP}

Q11. How much, if at all, do you think your age will affect your chances of getting a new job?

\begin{tabular}{|c|c|c|c|}
\hline ALL & $\begin{array}{c}\text { NET } \\
\text { Ages 50-65 }\end{array}$ & Ages 50-59 & Ages 60-65 \\
\hline Weighted Base & 861 & 623 & 237 \\
\hline Very/Somewhat helpful (Net) & $12 \%$ & $13 \%$ & $7 \%$ \\
\hline My age will be very helpful (5) & $5 \%$ & $5 \%$ & $4 \%$ \\
\hline My age will be somewhat helpful (4) & $7 \%$ & $8 \%$ & $2 \%$ \\
\hline $\begin{array}{l}\text { My age will neither help nor hurt my } \\
\text { chances of getting a new job (3) }\end{array}$ & $26 \%$ & $28 \%$ & $21 \%$ \\
\hline Very/Somewhat Hurtful (Net) & $62 \%$ & $59 \%$ & $72 \%$ \\
\hline My age will be somewhat hurtful (2) & $42 \%$ & $44 \%$ & $38 \%$ \\
\hline My age will be very hurtful (1) & $20 \%$ & $15 \%$ & $34 \%$ \\
\hline
\end{tabular}

Q12. How interested are you in receiving help in your job search process?

\begin{tabular}{|l|c|c|c|}
\hline ALL & $\begin{array}{c}\text { NET } \\
\text { Ages 50-65 }\end{array}$ & Ages 50-59 & Ages 60-65 \\
\hline Weighted Base & $\mathbf{8 6 1}$ & $\mathbf{6 2 3}$ & $\mathbf{2 3 7}$ \\
\hline Very/Somewhat interested (Net) & $68 \%$ & $72 \%$ & $57 \%$ \\
\hline Very interested (4) & $24 \%$ & $26 \%$ & $18 \%$ \\
\hline Somewhat interested (3) & $44 \%$ & $46 \%$ & $39 \%$ \\
\hline Not too/Not at all interested (Net) & $32 \%$ & $28 \%$ & $43 \%$ \\
\hline Not too interested (2) & $22 \%$ & $19 \%$ & $28 \%$ \\
\hline Not at all interested (1) & $10 \%$ & $9 \%$ & $15 \%$ \\
\hline
\end{tabular}

Q13a. Sometimes people stop looking for work because of difficulty finding a job. How seriously are you considering ending your job search because of difficulty finding a job?

\begin{tabular}{|l|c|c|}
\hline & $\begin{array}{c}\text { NET } \\
\text { Ages 50-65 } \\
\text { UNEMPLOYED }\end{array}$ & Ages 50-59 \\
\hline Weighted Base & $\mathbf{1 2 8}$ & Insufficient base \\
\hline Very/Somewhat seriously (Net) & $39 \%$ & \\
\hline Very seriously (4) & $8 \%$ & \\
\hline Somewhat seriously (3) & $30 \%$ & \\
\hline Not too/Not at all seriously (Net) & $61 \%$ & \\
\hline Not too seriously (2) & $34 \%$ & \\
\hline Not at all seriously (1) & $27 \%$ & \\
\hline
\end{tabular}




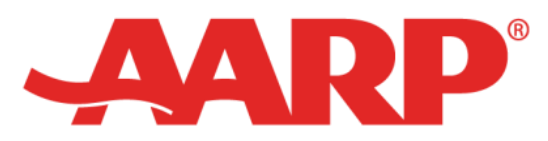

Q13b. Sometimes people decide not to look for work because of difficulty finding a job. If you lost your job within the next year, how seriously would you consider NOT looking for a new job?

\begin{tabular}{|c|c|c|c|}
\hline EMPLOYED & $\begin{array}{c}\text { NET } \\
\text { Ages 50-65 } \\
\text { EMPLOYED }\end{array}$ & Ages 50-59 & Ages 60-65 \\
\hline Weighted Base & 733 & 540 & 193 \\
\hline Very/Somewhat seriously (Net) & $37 \%$ & $35 \%$ & $45 \%$ \\
\hline Very seriously (4) & $13 \%$ & $12 \%$ & $18 \%$ \\
\hline Somewhat seriously (3) & $24 \%$ & $23 \%$ & $27 \%$ \\
\hline Not too/Not at all seriously (Net) & $63 \%$ & $66 \%$ & $55 \%$ \\
\hline Not too seriously (2) & $28 \%$ & $27 \%$ & $31 \%$ \\
\hline Not at all seriously (1) & $35 \%$ & $39 \%$ & $24 \%$ \\
\hline
\end{tabular}

